\title{
Programas de formación para el apoyo entre iguales en salud mental en España
}

\section{Training programs for peer support in mental health in Spain}

\author{
Rebeca Zabaleta González¹, Fernando Lezcano Barbero²
}

\begin{abstract}
y María Victoria Perea Bartolomé 3
${ }^{1}$ Contratada predoctoral. Departamento de Ciencias de la Educación, Universidad de Burgos, Burgos, España. E-mail: rzabaleta@ubu.es

${ }^{2}$ Profesor Titular de Universidad. Departamento de Ciencias de la Educación, Universidad de Burgos, Burgos, España. E-mail: flezcano@ubu.es

${ }^{3}$ Catedrática de Universidad. Departamento de Psicología Básica, Psicobiología y Metodología de las Ciencias del Comportamiento, Universidad de Salamanca, Salamanca, España.

E-mail: vperea@usal.es
\end{abstract}

El trabajo que se presenta ha sido financiado por la Universidad de Burgos (contrato predoctoral 2018) y por la Fundación Banco Sabadell (Ayudas a la investigación 2018).

Los autores agradecen la colaboración de todas las personas y organizaciones que han facilitado información sobre sus experiencias para que esta investigación sea posible.
Departamento de Ciencias de la Educación,
Universidad de Burgos.
Burgos, España.

\section{Resumen}

Los problemas de salud mental son un tema relevante en todo el mundo. Pese a los avances, es necesario continuar trabajando para mejorar la calidad de vida del colectivo. La educación y el apoyo entre iguales son estrategias muy adecuadas para contribuir a su integración socio-laboral. Se ha realizado una revisión bibliográfica en bases de datos y páginas web para identificar experiencias españolas de formación de personas con Trastorno Mental Severo (TMS) basadas en el apoyo entre iguales. Se han encontrado siete experiencias de formación para el colectivo, cinco de ellas centradas en el apoyo entre iguales y otras en la figura de el/la asistente personal.

El número de experiencias encontradas en España es muy reducido. Aunque los objetivos son comunes, se observan enfoques diferentes (entorno sanitario, laboral, social) que pueden ser muy relevantes para el diseño y aplicación de otras experiencias en diferentes contextos. Palabras clave: salud mental, enfermedad mental, formación, apoyo entre iguales, educación.

\section{Abstract}

Mental health problems are a relevant issue all around the world. Mental illness affects one in four people throughout their lives, causing serious socio-economic and human rights-related consequences.

In Spain, despite the progress made with psychiatric reform and the promotion of a community model, it is necessary to continue working to improve the quality of life of people 
with mental health problems. Education and peer support are highly recommended strategies to contribute to their social and occupational integration.

Peer support is a relationship based on mutual support. It is established between two people: the person with mental health problems trained to provide the support and the person who receives it. This support enhances the recovery process. For this support to be effective, it is essential to previously train the person who will undertake the counselling functions.

The relationship of support offers practical advice, suggestions, strategies and emotional and psychological support. In addition, maintaining an non-professional perspective is essential to help people to rebuild their sense of community after an unknown experience. Peer support brings benefits to all parties involved: support providers, receivers and implementing organizations or institutions. However, training programs for peer support are not widespread.

In Spain, despite the recognition of the need to integrate people with mental health problems into society and to respond to their needs, there is no published research on guidelines for such training programs.

For this reason, this research identifies the peer support training programs which have been or are being carried out in Spain and describe their formal characteristics.

A bibliographic review of databases and web pages has been undertaken to identify Spanish experiences of training people with Severe Mental Disorder (SMD) based on peer support and to describe their formal characteristics (duration, methodology, evaluation and workplace).

A total of 77 programs found in the electronic databases, 75 of which have been excluded as they are not Spanish experiences. Five programs were found in the searches carried out on the reports of conferences, congresses and websites of Spanish organizations and institutions. Finally, seven training experiences were selected for the group, five of them focused on peer support and others on the figure of the personal assistant. The study of the programs is carried out following the indications of the content analysis model.

The training programs described have common objectives, however, they vary considerably in their approach, duration, names of support and evaluation providers and, to a lesser extent, in the contents, methodology and workplaces.

Despite the expansion of peer support in mental health, the number of experiences found in Spain is limited. Any program must be adapted to the strengths of the individuals, the type of work, as well as to the needs of the community. Furthermore, if this new professional profile is regulated at work, it will contribute to improving the rates of social and occupational integration of people with mental health problems.

It is necessary to continue developing and implementing experiences of these characteristics and to investigate their efficiency and effectiveness in order to be able to provide guidelines on peer support in the Spanish population.

Keywords: mental health, mental illness, training, peer support, education.

\section{Introducción}

La salud mental es un tema relevante para todos, puesto que se relaciona con el desarrollo de las sociedades y los países (OMS, 2008). Según la Organización Mundial de la Salud (OMS, 2017a) su prevalencia continúa aumentando, provocando considerables efectos en las personas, consecuencias graves a nivel socioeconómico y en los derechos humanos de las personas en todos los países.

La misma organización (OMS, 2004) estimó que las enfermedades mentales afectan a unos 450 millones de personas en todo el mundo. Estas suponen el $40 \%$ de las enfermedades crónicas y la mayor causa de los años vividos con discapacidad. Una de cada cuatro personas padece alguna enfermedad mental a 
lo largo de su vida (Ministerio de Sanidad y Consumo, 2007; OMS, 2001; OMS, 2015).

Las enfermedades mentales incluyen un amplio conjunto de patologías que, si bien tienen elementos comunes, presentan enormes diferencias. Por ello, esta investigación se centrará en los trastornos más graves, que afectan durante un periodo prolongado. Para referirse a ellos se empleará el término "trastorno mental severo" (TMS), propuesto por la AEN (Asociación Española de Neuropsiquiatría, 2003; OMS, 2017b), que cuenta con gran consenso científico (López, 2008; Rodríguez, 2007).

Este grupo de población presenta una problemática común en relación a la discapacidad funcional y social y la necesidad de recuperación. Estos problemas se concretan en cada persona, de manera particular e individualizada en función de la interacción de diversos factores y requieren diferentes niveles de atención e intervención (AEN, 2003; Ruiz et al., 2012).

A pesar de que cada TMS se manifiesta de forma diferente en cada persona, presentan dificultades y necesidades específicas vinculadas a los problemas psiquiátricos y psicosociales (AEN, 2003; López y Laviana, 2017):

- Atención y tratamiento de la salud mental: apoyo personal continuado, intervenciones biológicas y psicológicas, psicoterapia, rehabilitación cognitiva.

- Atención en crisis.

- Rehabilitación psicosocial y apoyo a la integración social: entrenamiento en habilidades y desarrollo de capacidades, soportes o apoyos específicos.

- Rehabilitación laboral y apoyo a la integración en el mundo del trabajo.

- Alojamiento y atención residencial comunitaria.

- Apoyo económico.

- Apoyos no profesionales (del entorno familiar y de las redes sociales).

- Protección y defensa de sus derechos: defensa jurídica e institucional.

- Apoyo a las familias.
Es necesario un marco normativo/legislativo para proteger los derechos de las personas con trastornos mentales. Según el informe de la OMS (2010) Salud mental y desarrollo: poniendo a las personas con problemas de salud mental con un grupo vulnerable, las personas con dificultades de salud mental satisfacen los principales criterios de vulnerabilidad (Funk et al., 2010).

En 2017, la OMS impulsó la iniciativa Quality Rights con el objetivo de mejorar la calidad de la atención en salud mental y los servicios relacionados, además de promover los derechos de las personas con discapacidad (psicosociales, intelectuales y cognitivas) alrededor del mundo (OMS, 2017). Mediante Quality Rights, la OMS está apoyando a los países para establecer políticas, estrategias, derechos y servicios que estén acorde con los estándares internacionales de derechos humanos (OMS, 2018). Uno de estos derechos fundamentales es la educación, ya que va a posibilitar su participación ciudadana en los diferentes ámbitos (económico, cultural y político). Pero existe una falta de definición sobre el papel que ocupa la educación en la salud mental (García-Pérez, Peña-Calvo y Torío-López, 2016; Pié, 2000).

En España, a pesar del esfuerzo cooperativo de profesionales de la psiquiatría, la salud mental y el derecho, no se dispone de una legislación específica para las personas con problemas de salud mental (González, 2009; Ministerio de Sanidad y Consumo, 2007). Destacamos la Ley 26/2011, del $1 .^{\circ}$ de agosto, de adaptación normativa a la Convención Internacional sobre los Derechos de las Personas con Discapacidad, en la que se adecúa la regulación legislativa sobe discapacidad siguiendo las directrices de la Convención Internacional de los Derechos de las Personas con Discapacidad (2006). Además, en el Real Decreto Legislativo 1/2013, del 29 de noviembre, por el que se aprueba el Texto Refundido de la Ley General de derechos de las personas con discapacidad y de su inclusión social, se diferencia la discapa- 
cidad mental dentro de la categoría jurídica de discapacidad (De Fuentes, 2016).

La reforma psiquiátrica en España deriva de la Ley General de Sanidad (Ley 14/86) y su desarrollo concreto en las diferentes Comunidades Autónoma. El Capítulo III: De la salud mental, artículo 20, de la Ley General de Sanidad (Ley 14/86 de 25 de abril) contempla la integración de los servicios psiquiátricos en el sistema sanitario general y la total equiparación de los enfermos mentales al resto de personas que requieran servicios asistenciales y sociales. Esta nueva articulación del Sistema Nacional de Salud promueve la descentralización del estado con la progresiva transferencia de las competencias en sanidad a las Comunidades Autónomas, "respondiendo a la idea de proximidad de los servicios a los usuarios y de gestión descentralizada y participativa" (Ley 14/86 de 25 de abril). Esta desvinculación permite a la ley hacer solo una declaración de principios en temas clave como la integración en el sistema sanitario general o el desarrollo de programas y servicios de rehabilitación e integración social, provocando que las estructuras de atención (organización, recursos y gestión) sean muy diversas en las diferentes comunidades (López y Laviana, 2017).

El acercamiento de la salud mental a la población en general con el modelo comunitario supuso el fin de los muros tangibles pero no de las barreras imaginarias y el estigma que impiden la plena integración del colectivo, por lo que debemos seguir trabajando en ello (Arnaiz y Uriarte, 2006; Madoz-Gúrpide et al., 2017). El estigma hace referencia a los estereotipos, prejuicios y la discriminación que sufre un grupo (Arnaiz y Uriarte, 2006), en este caso, las personas con enfermedad mental y se debe principalmente a conceptos erróneos generalizados sobre las causas y la naturaleza de las enfermedades mentales (OMS, 2010). El estigma afecta la capacidad para realizar actividades de la vida diaria y es una de las barreras más importantes en el acceso a tratamientos efectivos (Moreno y Desviat, 2012; Naslund et al., 2016). Además, esta discriminación y exclusión provoca, en muchas ocasiones, que las personas con enfermedad mental internalicen un sentimiento de culpa causando una percepción negativa sobre sí mismos, que se puede sintetizar con el término "autoestigma". Esto dificulta su autodefensa y el pleno ejercicio de sus derechos (Torras, 2012). Según López et al. (2008), para hacer frente a los estereotipos, son necesarios programas educativos que incluyan no solo información, sino discusión, casos reales $\mathrm{y}$, especialmente, presencia de personas con problemas de salud mental.

Durante la reforma psiquiátrica se crean las primeras asociaciones específicas para personas con enfermedad mental. En España, el movimiento asociativo comenzó a surgir en el año 1968 en diferentes lugares del estado (Ariño y San Pío, 2007; Confederación Salud Mental España, 2017). El movimiento de usuarios de salud mental tiene su origen hace menos de 20 años, liderado por representantes de asociaciones de usuarios o familiares. El desarrollo ha sido desigual en los diferentes territorios y muchas asociaciones tienen un carácter local (Ministerio de Sanidad, Política Social e Igualdad, 2011; Torrás, 2012).

Desde la dimensión socioeducativa, se trata de dotar de los recursos necesarios para incrementar su calidad de vida e inclusión social (García-Pérez et al., 2017). La educación es uno de los elementos que va a favorecer el bienestar y la satisfacción con la vida (Hernández y Carranza, 2017).Entre otros, existen programas de apoyo social que tratan de facilitar a las personas con TMS la posibilidad de desarrollar una vida comunitaria ayudándoles a cubrir determinadas necesidades básicas (López, Fernández y Laviana, 2010).

La investigación ha demostrado que el apoyo social influye en diversos aspectos relacionados con los procesos de salud y enfermedad (Barrón y Sánchez, 2001). Es por ello que el apoyo social es importante, ya que permite el mantenimiento de la integridad psicológica y física de la persona, y 
son sus funciones principales aumentar las capacidades personales y promover el logro de las metas vitales de cada persona. En el caso de las personas con TMS, el apoyo social se relaciona positivamente con el estado de salud y con la recuperación de la enfermedad. Del mismo modo, la ausencia o carencia de apoyo social se puede considerar un factor de riesgo, que se puede relacionar con la aparición de enfermedades mentales. (Barra, 2004; Vega-Angarita y González-Escobar, 2017). Es por la relación de los programas de apoyo social con las entidades sociales para personas con problemas de salud mental que en este trabajo se usa el término "apoyo mutuo".

A comienzos de la década de 1980, Gartner y Riessman (1982) definieron el apoyo mutuo entre personas con problemas de salud mental como la ayuda ofrecida entre personas con problemas similares y con la finalidad de lograr un deseado cambio social o personal. La definición más extendida de apoyo mutuo, desde principios del siglo XXI, es la de Mead, Hilton y Curtis (2001) que lo definen como un sistema de dar y recibir ayuda basado en los principios clave de respeto, responsabilidad compartida y acuerdo mutuo de lo que es útil. Para que esta ayuda sea efectiva es importante formar previamente a la persona que cumpla la función de asesoramiento. Además, experiencias internacionales demuestran (Bono del Trigo, Navarro y Mena, 2011) que el carácter formal otorga un valor añadido: (1) para el agente de ayuda mutua supone un empoderamiento y mejora de la autoestima, así como el desarrollo de habilidades de comunicación, emocionales y sociales; y (2) para el receptor de esta ayuda, dado que es una oportunidad de sentirse escuchado y comprendido, de expresarse con más libertad y confianza en la relación con un igual para finalmente incrementar su funcionamiento social y autoestima.

Una relación concreta del apoyo mutuo que se da entre pares o iguales (Smith y Bradstreet, 2011). Se trata de una práctica cuya finalidad es aprovechar las fortalezas para promover y proporcionar cualquier soporte que las personas requieran para ayudarles a moverse más cerca de alcanzar sus ambiciones, y se basa fundamentalmente en la empatía (Watson, Lambert y Machin, 2016).

El apoyo de pares ha sido planteado como una forma de promover la recuperación para cualquier persona que ha tenido dificultades de salud mental. Por ejemplo, mediante la motivación de la autoeficacia y la esperanza a través del intercambio de conocimiento experiencial y a través del modelado de estrategias de recuperación y afrontamiento (Lloyd-Evans et al., 2014). En esta propuesta formativa se ofrecen consejos prácticos, sugerencias, estrategias y apoyo emocional y psicológico. Este apoyo se reconoce como un importante facilitador en la recuperación. Mantener un punto de vista no profesional es crucial para ayudar a las personas a reconstruir su sentido de comunidad tras una experiencia desconocida (Campos et al., 2016; Gillard y Holley, 2014; Intentional Peer Support, 2017; Mead y MacNeil, 2006).

Los servicios de apoyo entre iguales se han extendido a lo largo del mundo, ya que se ha demostrado que son efectivos para mejorar la vida de las personas con dificultades de salud mental y abuso de sustancias. Los inicios del apoyo entre iguales comenzaron en Estados Unidos, donde desempeñaron un papel clave durante la transformación de los servicios tradicionales en servicios de salud mental basados en la comunidad (ENIL, 2018; University of Nebraska, 2003).

Respecto a Europa, los programas de formación para el apoyo entre iguales no están generalizados, ya que el apoyo mutuo y su importancia no son bien conocidos, especialmente para las personas con enfermedad mental, y se carece de fondos estatales (ENIL, 2018). En España ya está reconocida la necesidad de integrar a las personas con dificultades de salud mental en la sociedad y responder de manera adecuada y eficaz a sus necesidades. Pero, hasta la fecha, no existe ninguna investigación publicada sobre las directrices para el apoyo entre iguales en situaciones de salud 
mental en una población española (Campos et al., 2016).

\section{Método}

Conociendo la importancia del apoyo entre iguales en salud mental y la necesidad de formar previamente a la persona que va a proveer el apoyo, ¿qué características tienen los programas de formación que se llevan a cabo en España? El objetivo de este trabajo es identificar las experiencias de formación de pares para personas con trastorno mental severo que se han llevado, o se están llevando a cabo, en España y describir sus características formales.

El criterio principal de elegibilidad será que los programas de apoyo entre iguales estén implementados en España. El diferente grado de desarrollo de este tipo de programas en cada uno de los países hace difícil la comparación. Así, por ejemplo, en Estados Unidos, la mayoría de los estados tienen programas de estas características, sistemas de acreditación, certificación y revisión. Esta realidad es escasamente comparable con la realidad de España y de países ajenos a esa cultura. Asimismo, se excluyen los programas de voluntariado y de apoyo de profesionales a personas con enfermedad mental, por lo que se selecciona exclusivamente el apoyo entre iguales.

El sistema es el análisis de contenido siguiendo las indicaciones de Green et al. (2007) que indica los siguientes pasos: inmersión en el texto, codificación, creación de categorías y generación de temas. En este trabajo, los temas serán las distintas características que definen cada uno de los programas y para ello, se ha realizado:

1) Revisión bibliográfica internacional en bases de datos electrónicas (EROC, WOS o SSCI). Se han usado palabras clave como: "Formación y apoyo entre iguales"; "apoyo mutuo" y "Trastorno Mental Severo" / "salud mental" / "enfermedad mental", tanto en español como en inglés ("Training and peer support"; "mutual support and Severe Mental Disorder" / "mental health" / "mental illness").

2) Se han revisado actas de congresos y jornadas, las páginas web de organizaciones sociales relacionadas con la salud mental (asociaciones, fundaciones, federaciones y confederaciones) y otros organismos (Ministerio de Salud, servicios de salud mental).

3) Se ha contactado vía e-mail y/o telefónicamente a los responsables implicados en las experiencias para recabar más información de los programas (duración, metodología, evaluación y lugar de empleo) o servicios cuando la información aportada en los artículos o sitios web no fuera suficiente.

En un primer momento, en las búsquedas realizadas en las bases de datos electrónicas, se encontraron 77 programas de formación para el apoyo entre iguales, de los cuales se descartaron 75 porque no se implementan en España. Posteriormente, se llevaron a cabo las búsquedas en actas de jornadas, congresos y búsquedas en webs de organizaciones y organismos españoles y se localizaron otras cinco experiencias. Finalmente se seleccionaron para su análisis un total de siete programas de formación de pares para personas con TMS en España. Entre las limitaciones del estudio se observa que pueden existir otras experiencias que no han sido identificadas al no estar publicadas, pues en el ámbito de las organizaciones sociales no está instaurada la cultura de la publicación de investigaciones y/o experiencias y no están accesibles para los/as investigadores/as.

\section{Resultados}

Como se ha mencionado anteriormente, España está iniciándose en esta práctica formativa. Organizaciones sociales y sanitarias de este país han participado en proyectos europeos de formación de pares (Proyecto EMILIA y Peer2Peer) durante los años 2005 y 2015, respectivamente, que fueron el embrión 
de la realidad actual. A continuación se detallan los aspectos más relevantes de cada uno de los programas encontrados.

\section{Proyecto EMILIA}

El primer proyecto europeo en el que participó España fue el Proyecto EMILIA (Empowerment of Mental Illness Service Users: Lifelong Learning, Integration and Action) implementado entre septiembre de 2005 y octubre de 2010 (Palomer et al., 2011). Equipos de salud mental del Hospital del Mar y del Centre Fòrum en Barcelona participaron en esta experiencia pionera en la formación del usuario experto. El objetivo del proyecto es reducir la exclusión social de la población con TMS a través de la formación continuada. La formación consta de 10 sesiones, con una duración de 1 hora y 45 minutos cada una.

La metodología sigue el modelo life long learning (LLL) basado en el aprendizaje por la experiencia. Los contenidos que se trabajan son: a) la red de soporte social, la recuperación y la interacción; b) las fortalezas personales y c) el plan de desarrollo personal. A esta formación se incluye un taller multifamiliar. El proyecto se enmarca en un entorno sanitario/hospitalario.

Como resultados de la formación se abrieron dos líneas de implicación laboral (Palomer et al., 2010):

El usuario como docente colaborador y formador de otros usuarios y

El usuario como mediador entre las instituciones sanitarias

El principal beneficio obtenido es la mejora de la respuesta aportada a las personas usuarias de los centros donde se desarrolla esta práctica.

\section{Proyecto Peer2Peer}

El proyecto Peer2Peer (P2P) es un curso de formación financiado por la Unión Europea (UE) y enmarcado en el Programa de Aprendizaje Permanente "Leonardo Da Vinci". P2P es una adaptación del programa Professional Development Award (PAD) in Mental Health Peer Support desarrollado e implementado previamente por el Scottish Recovery Network (Fundación INTRAS et al., 2015). El objetivo es formar a personas con problemas de salud mental para que trabajen como agentes de apoyo mutuo. Son personas que han superado una enfermedad mental y que se convertirán en referentes para otras personas que estén atravesando una dificultad de salud mental (Orihuela, Conde y Marcos, 2015).

El curso tiene una duración de 48 horas distribuido en 12 sesiones que se plantean de forma práctica y participativa. Como punto de partida, en todas ellas, se usa la propia experiencia de las personas. Se emplean estrategias educativas diversas: dinámicas de grupo, debates, psicodrama, video terapia, roleplaying, actividades dirigidas por el dinamizador. Los principales contenidos que se abordan son: (1) el apoyo mutuo; (2) la recuperación; y (3) el desarrollo de habilidades necesarias para el desempeño como agente de apoyo mutuo. Respecto a la evaluación, se lleva a cabo una evaluación intermedia y otra final. Las organizaciones españolas participantes son: Fundación INTRAS (Valladolid), Fundación en Primera Persona (Andalucía) y un miembro de la asociación AGIFES (Gipuzkoa).

Los receptores de la formación desempeñan sus funciones como agentes de apoyo mutuo en entornos sanitarios de forma voluntaria, puesto que no hay constancia todavía de que se haya formalizado un contrato laboral.

\section{Proyecto Ayuda Mutua en los servicios de salud mental de Andalucía}

Andalucía ha sido la comunidad autónoma pionera que ha llevado a cabo la primera formación de pares implementada exclusivamente en España en 2010-2011 a través del Proyecto Ayuda Mutua en los servicios de salud mental de Andalucía. El proyecto se enmarca en el II Plan Integral de Salud Mental de Andalucía y ha sido coordinado por la Escuela Andaluza 
de Salud Pública y la Federación Andaluza de Asociaciones de Personas con Enfermedad Mental. El curso tiene como objetivo formar a personas con problemas de salud mental, para actuar como agentes de ayuda mutua y la realización de sesiones de ayuda mutua (entre un agente y un par receptor) en los servicios de salud mental de Andalucía (Bono del Trigo et al., 2011).

La formación se compone de 32 horas lectivas y 8 horas no presenciales. Utiliza una metodología participativa (debates, actividades en grupo, juegos de rol, etcétera) apoyada por presentaciones teóricas y síntesis integrativas de los contenidos. Posteriormente, con algunos de los participantes se realizó un curso de profundización de 12 horas enfocado al desarrollo de las sesiones de ayuda mutua.

Los agentes de apoyo mutuo desempeñan sus funciones en unidades de gestión clínica de los servicios de salud mental de Andalucía (unidades de salud mental comunitaria, hospitales de día y unidades de rehabilitación de salud mental).

\section{Expertos por experiencia}

El Gobierno de Castilla-La Mancha, a través de la Fundación Sociosanitaria de Castilla-La Mancha, y con el trabajo conjunto de las consejerías de Economía, Empresas y Empleo y la de Sanidad, ha desarrollado un proyecto de "expertos por experiencia". El objetivo es integrar a personas con problemas de salud mental dentro del equipo de los Centros de Rehabilitación Psicosocial y Laboral (CRPSL) para trabajar como profesionales. Su actividad profesional se desarrolla compartiendo sus vivencias y poniendo en valía sus fortalezas, asesorando a profesionales y realizando actividades de formación y sensibilización a otros (usuarios, familiares, profesionales).

El proyecto se inició en el 2018. La formación se desarrolla en dos fases:

- Se inicia con una formación interna de 11 sesiones de cinco horas cada una, donde se trabajaron contenidos como: (1) recuperación y apoyo mutuo; (2) tareas y el rol del experto por experiencia; (3) Plan de Bienestar Personal (PARB); (4) derechos. Para ello, se construyen de forma conjunta las actividades, priorizando siempre las capacidades, preferencias y particularidades de las personas y la realidad concreta de cada equipo y recurso.

- Posteriormente se realiza una supervisión de los expertos por experiencia, contratados en los CRPSL, a través de la revisión de temas concretos de su día a día.

Al momento de la redacción del presente trabajo, se sabía de la contratación de ocho expertos por experiencia. Es la primera comunidad autónoma en insertar laboralmente esta figura profesional (Casas et al., 2017).

El proyecto ha tenido un impacto muy positivo en los profesionales expertos por experiencia, en los receptores del apoyo mutuo y en los centros de rehabilitación psicosocial y laboral en los que se ha desarrollado; todo ello se ha constatado en las valoraciones realizadas por los diferentes agentes que han participado en las experiencias.

\section{Informando en primera persona}

La Confederación Salud Mental de España desde 2015 ha realizado en dos ediciones el curso Informando en primera persona. El objetivo es dotar a las personas con enfermedad mental de información y herramientas para que puedan hacer una labor de acogida y acompañamiento a otras personas usuarias en las entidades a las que pertenecen (Confederación Salud Mental España, 2017).

El curso tiene una duración de 10 horas que se lleva a cabo en dos días. Se desarrolla a través de estrategias educativas como: debates, reflexión y dinámicas grupales. Los contenidos trabajados son: (1) las habilidades de comunicación; (2) los derechos de las personas con enfermedad mental; y (3) 
la participación. La evaluación, al finalizar la formación, se realiza a través de un cuestionario. Seis meses después, se aporta otro cuestionario para conocer la valoración de su utilidad pasado el tiempo.

\section{Curso de asistente personal}

Se señala también el Curso de asistente personal, que se lleva a cabo desde 2016 por la Federación Salud Mental Castilla y León (y las 11 asociaciones miembro). Se trata de una formación y capacitación laboral teórico-práctica específica para personas con problemas de salud mental siguiendo el modelo de apoyo de pares (Robles, 2017).

La duración del curso varía de 50 a 250 horas de formación, donde los participantes a través de teoría y práctica, adquieren los conocimientos y habilidades necesarias para desenvolverse como asistentes personales de otras personas. Al terminar la formación se lleva a cabo una evaluación final.

Aunque la asistencia personal no se basa solo en el modelo del apoyo entre iguales, es interesante señalar esta formación destinada a personas con problemas de salud mental que sigue la idea del apoyo entre iguales.

\section{Programa ¡ACOMPAÑAME!}

Finalmente, ¡ACOMPAÑAME! es un programa de formación para personas con problemas de salud mental que se encuentran en un proceso de recuperación y que participan en una organización social. Su objetivo es formar en ayuda mutua y apoyo entre iguales a usuarios con problemas de salud mental para que actúen como compañeros de apoyo en la recepción, proceso de incorpora- ción y adaptación de nuevas personas que se unen a la entidad, así como en el acompañamiento en su recuperación.

La formación se compone de un total 12 sesiones, con una duración entre 90 y 120 minutos cada una, donde se desarrollan actividades de diversa índole (teóricas, de roleplaying, debates, reflexión) y se abordan contenidos relacionados con: (1) la recuperación; (2) el apoyo entre iguales; (3) habilidades de comunicación y de escucha. Como elementos más novedosos el curso incluye la realidad virtual y la robótica. Además, se realizan dos sesiones adicionales de evaluación: una inicial, donde se evalúa la situación cognitiva de las personas participantes a través de tres pruebas psicométricas y se formaliza su participación en el programa. Una final en la que se tiene en cuenta la información aportada durante la evaluación continua de la formación. Se realiza un retest de las pruebas psicométricas de la evaluación y se desarrollan pruebas específicas de evaluación: test de conocimientos, pruebas de role playing $\mathrm{y}$ estudio de casos.

También se lleva a cabo una evaluación del programa, a través de la valoración de las actividades después de cada sesión y unos cuestionarios finales (para el dinamizador y las personas participantes).

El programa, que ha sido validado por un panel internacional de expertos, ha comenzado a implementarse en mayo del 2019 (en entidades de Madrid, Burgos y Navarra) y se encuentra en su fase final de evaluación.

En la Tabla 1 se describen las principales características formales (perfil del proveedor del apoyo, duración, contenidos, metodología, evaluación, lugar de empleo y página web) de cada programa. 
Tabla 1

Experiencias formativas de pares en España.

\begin{tabular}{|c|c|c|c|c|c|c|c|}
\hline Programa & Perfil & Duración & Contenidos & $\begin{array}{c}\text { Metodología } \\
\text { (estrategias } \\
\text { educativas) }\end{array}$ & Evaluación & Empleo & Web \\
\hline $\begin{array}{l}\text { Proyecto } \\
\text { Emilia } \\
\text { (Barcelona) }\end{array}$ & $\begin{array}{l}\text { Usuario } \\
\text { experto }\end{array}$ & $\begin{array}{c}10 \text { sesiones } \\
(1.45 \mathrm{~h} / \mathrm{s})\end{array}$ & $\begin{array}{l}\text { Taller multifa- } \\
\text { miliar, red de } \\
\text { soporte social, } \\
\text { recuperación } \\
\text { e interacción, } \\
\text { fortalezas, plan } \\
\text { de desarrollo } \\
\text { personal }\end{array}$ & $\begin{array}{l}\text { Lifelong lear- } \\
\text { ning, aprendi- } \\
\text { zaje basado en } \\
\text { la experiencia }\end{array}$ & & $\begin{array}{l}\text { Entorno } \\
\text { sanitario }\end{array}$ & $\begin{array}{l}\text { http://bit. } \\
\text { ly/2lnsqXX }\end{array}$ \\
\hline $\begin{array}{c}\text { Peer2Peer } \\
\text { (Valladolid, } \\
\text { Andalucía y } \\
\text { Gipuzkoa) }\end{array}$ & $\begin{array}{c}\text { Agentes } \\
\text { de apoyo } \\
\text { mutuo }\end{array}$ & $\begin{array}{l}48 \text { horas, } \\
12 \text { sesiones }\end{array}$ & $\begin{array}{l}\text { Recuperación y } \\
\text { apoyo mutuo, } \\
\text { habilidades } \\
\text { de valores } \\
\text { del agente de } \\
\text { apoyo mutuo }\end{array}$ & $\begin{array}{l}\text { Aprendizaje } \\
\text { participativo, } \\
\text { análisis y } \\
\text { reflexión, teoría } \\
\text { y práctica. } \\
\text { Psicodrama y } \\
\text { video terapias. } \\
\text { Dinámicas de } \\
\text { grupo, debates, } \\
\text { role playing, } \\
\text { actividades } \\
\text { dirigidas por el } \\
\text { facilitador. }\end{array}$ & $\begin{array}{l}\text { Evaluación } \\
\text { intermedia y } \\
\text { final }\end{array}$ & $\begin{array}{c}\text { Entorno } \\
\text { sanitario }\end{array}$ & $\begin{array}{l}\underline{\mathrm{http}: / / \mathrm{bit} .} \\
\underline{\text { ly/2BXvGl1 }}\end{array}$ \\
\hline $\begin{array}{l}\text { Proyecto } \\
\text { Ayuda } \\
\text { Mutua en } \\
\text { los servicios } \\
\text { de salud } \\
\text { mental de } \\
\text { Andalucía }\end{array}$ & $\begin{array}{c}\text { Agentes } \\
\text { de ayuda } \\
\text { mutua }\end{array}$ & $\begin{array}{c}32 \mathrm{~h} \\
\text { lectivas, } \\
8 \mathrm{~h} \text { no } \\
\text { presen- } \\
\text { ciales }+ \\
12 \mathrm{~h} \text { curso } \\
\text { profun- } \\
\text { dizar }\end{array}$ & $\begin{array}{l}\text { Herramientas } \\
\text { de Comunica- } \\
\text { ción, Recupe- } \\
\text { ración, Ayuda } \\
\quad \text { Mutua }\end{array}$ & $\begin{array}{l}\text { Muy partici- } \\
\text { pativa, con } \\
\text { actividades de } \\
\text { debate, trabajo } \\
\text { en grupos o } \\
\text { juegos de roles, } \\
\text { apoyados con } \\
\text { breves intro- } \\
\text { ducciones } \\
\text { teóricas con } \\
\text { soporte audio- } \\
\text { visual y puesta } \\
\text { en común de } \\
\text { síntesis inte- } \\
\text { gradoras de los } \\
\text { contenidos. }\end{array}$ & $\begin{array}{c}\text { Cuestionario } \\
\text { autoaplicado. } \\
\text { Registro del } \\
\text { desempeño } \\
\text { (agente } \\
\text { y profe- } \\
\text { sionales). } \\
\text { Sesiones de } \\
\text { evaluación. }\end{array}$ & $\begin{array}{c}\text { Unidades } \\
\text { de Gestión } \\
\text { Clínica: U. } \\
\text { de Salud } \\
\text { Mental } \\
\text { Comu- } \\
\text { nitaria, } \\
\text { Hospitales } \\
\text { de Día } \\
\text { y U. de } \\
\text { Rehabilita- } \\
\text { ción Salud } \\
\text { Mental. }\end{array}$ & $\begin{array}{l}\underline{\text { http://bit. }} \\
\text { ly/2o3ugwN }\end{array}$ \\
\hline
\end{tabular}




\begin{tabular}{|c|c|c|c|c|c|c|c|}
\hline $\begin{array}{l}\text { Profesional } \\
\text { experto por } \\
\text { la expe- } \\
\text { riencia } \\
\text { (Castilla La } \\
\text { Mancha) }\end{array}$ & $\begin{array}{c}\text { Profe- } \\
\text { sionales } \\
\text { expertos }\end{array}$ & $\begin{array}{c}11 \text { sesiones } \\
(5 \mathrm{~h} / \mathrm{s})\end{array}$ & $\begin{array}{l}\text { Recuperación y } \\
\text { apoyo mutuo, } \\
\text { tareas y rol del } \\
\text { experto, Plan } \\
\text { de Bienestar } \\
\text { Personal } \\
\text { (PARB), dere- } \\
\text { chos, etc }\end{array}$ & $\begin{array}{l}\text { Construcción } \\
\text { conjunta de las } \\
\text { actividades, } \\
\text { priorizando las } \\
\text { capacidades, } \\
\text { preferencias y } \\
\text { particularidades } \\
\text { de las personas } \\
\text { y, la propia } \\
\text { realidad de } \\
\text { cada equipo y } \\
\text { recurso. }\end{array}$ & $\begin{array}{l}\text { Supervisión. } \\
\text { Revisión } \\
\text { de temas } \\
\text { concretos de } \\
\text { su día a día. }\end{array}$ & $\begin{array}{c}\text { Centros } \\
\text { de Reha- } \\
\text { bilitación } \\
\text { Psicosocial } \\
\text { y Laboral } \\
\text { (CRPSL) }\end{array}$ & $\begin{array}{l}\underline{\mathrm{https}: / / \mathrm{bit} .} \\
\underline{\mathrm{y} / 2 \mathrm{NImPHu}}\end{array}$ \\
\hline $\begin{array}{l}\text { In-formando } \\
\text { en primera } \\
\text { personas(Es- } \\
\text { paña) }\end{array}$ & $\begin{array}{l}\text { Apoyo } \\
\text { mutuo }\end{array}$ & 10 horas & $\begin{array}{l}\text { Habilidades } \\
\text { de comunica- } \\
\text { ción, derechos, } \\
\text { participación, } \\
\text { procesos }\end{array}$ & $\begin{array}{l}\text { Debate, } \\
\text { reflexión y } \\
\text { dinámicas } \\
\text { grupales }\end{array}$ & $\begin{array}{c}\text { Cuestionario } \\
\text { sobre la } \\
\text { formación. A } \\
\text { los } 6 \text { meses } \\
\text { cuestionario } \\
\text { utilidad }\end{array}$ & $\begin{array}{l}\text { Acogida y } \\
\text { acompa- } \\
\text { ñamiento } \\
\text { en las } \\
\text { entidades }\end{array}$ & $\frac{\text { https://bit. }}{\underline{\text { ly/2gNUy } 5 \mathrm{q}}}$ \\
\hline $\begin{array}{c}\text { Asistencia } \\
\text { personal } \\
(\mathrm{CyL})\end{array}$ & $\begin{array}{l}\text { Asistente } \\
\text { personal }\end{array}$ & $\begin{array}{c}\text { Varía } 50 \mathrm{~h}- \\
250 \mathrm{~h} .\end{array}$ & $\begin{array}{c}\text { El/la asistente } \\
\text { personal, } \\
\text { funciones, } \\
\text { modelos en } \\
\text { salud... }\end{array}$ & $\begin{array}{c}\text { Teoría y prác- } \\
\text { ticas }\end{array}$ & $\begin{array}{l}\text { Evaluación } \\
\text { final }\end{array}$ & $\begin{array}{c}\text { Asistencia } \\
\text { personal a } \\
\text { otros/as }\end{array}$ & $\frac{\text { https://bit. }}{\underline{\text { ly/2U3jVyk }}}$ \\
\hline $\begin{array}{l}\text { ¡Acompá- } \\
\text { ñame! }\end{array}$ & $\begin{array}{l}\text { Compa- } \\
\text { ñero/ } \\
\text { compa- } \\
\text { ñera de } \\
\text { apoyo }\end{array}$ & $\begin{array}{c}12 \text { sesiones } \\
(90-120 \\
\text { min cada } \\
\text { una })+2 \\
\text { sesiones } \\
\text { evaluación }\end{array}$ & $\begin{array}{c}\text { Recupera- } \\
\text { ción, apoyo } \\
\text { mutuo y apoyo } \\
\text { entre iguales, } \\
\text { habilidades de } \\
\text { comunicación, } \\
\text { de escucha, etc. }\end{array}$ & $\begin{array}{l}\text { Teoría, acti- } \\
\text { vidades } \\
\text { prácticas con } \\
\text { realidad virtual, } \\
\text { robótica, } \\
\text { role playing, } \\
\text { debates, } \\
\text { reflexión indi- } \\
\text { vidual, etc. }\end{array}$ & $\begin{array}{l}\text { Inicial (sit. } \\
\text { cognitiva), } \\
\text { continua y } \\
\text { final (sit. } \\
\text { Cognitiva, } \\
\text { estudio de } \\
\text { casos, role } \\
\text { playing, } \\
\text { conoci- } \\
\text { meintos) }\end{array}$ & $\begin{array}{l}\text { Entidades } \\
\text { sociales }\end{array}$ & $\begin{array}{l}\text { https://bit. } \\
\text { ly/36uugut }\end{array}$ \\
\hline
\end{tabular}

\section{Discusión}

En los últimos años se ha producido un rápido crecimiento y expansión del apoyo entre iguales en salud mental (Davidson y González-Ibáñez, 2017), aunque en la actualidad no existen estándares de educación reconocidos. Cualquier programa debe ser adaptado a las fortalezas de los individuos, el tipo de trabajo, así como a las necesidades de la comunidad (Behavioral Health Work Group of the NPSCLN, 2014; Chinmam et al., 2006).
En España se observa cómo los siete programas de formación descritos varían ampliamente en su duración, denominación de los proveedores del apoyo y evaluación y, en menor medida, en los contenidos, estrategias educativas y lugares de empleo. Del mismo modo, la formación y el desarrollo profesional varían ampliamente en otro países y es por ello que algunos han establecido programas formales de formación para los servicios de apoyo entre iguales (Behavioral Health Work Group of the NPSCLN, 2014). 
Los proveedores del apoyo pueden desempeñar un rol voluntario, sin compensación, o un rol laboral (Ostrow y Leaf, 2014). Es necesario, siguiendo el ejemplo de otros países y la experiencia mencionada en Castilla La Mancha, regular esta función profesional y que los trabajadores de apoyo entre iguales puedan desempeñar un puesto de trabajo regulado.

Prioritariamente, los proveedores del apoyo, desempeñan sus funciones en entornos sanitarios (Reppery Carter, 2011). En los programas señalados, a excepción de ¡Acompáñame!, el rol se enmarca en entornos sociosanitarios. Por lo que es preciso diseminar este perfil para que las entidades sociales sean conscientes de los importantes beneficios que aporta a las personas y a las organizaciones. Asimismo, es necesario invertir en salud mental (Tortella-Feliu et al., 2016) para, entre otras acciones, implementar este nuevo rol.

Puesto que estos programas de formación tienen un elevado potencial para el empleo y están reconocidos como importantes para mejorar las tasas de integración social del colectivo (Delman et al., 2014; Watson et al., 2016), su regulación laboral contribuiría a mejorar las tasas de inserción sociolaboral de las personas con problemas de salud mental. Además, las organizaciones laborales actúan como espacio formal de socialización donde la persona desarrolla habilidades para la ciudadanía (Lopera y Echeverri, 2018).

Estos programas son un ejemplo de buenas prácticas, un medio para promover los servicios centrados en la recuperación teniendo en cuenta la experiencia vivida y contribuir significativamente a mejorar los servicios de salud (Repper et al., 2013; Smith y Bradstreet, 2011; Watson et al., 2016).

\section{Conclusiones}

En España ya está reconocida, tanto en la legislación como en la práctica médica y en el entorno sociosanitario, la necesidad de integrar a las personas con problemas de salud mental en la sociedad y responder de manera adecuada y eficaz a sus necesidades.

Existen diferentes experiencias de formación de pares en España, pero su número es muy reducido si lo comparamos con otros países. En América del Norte, pioneros en estos programas, la mayoría de estados disponen de una formación, acreditación y puestos de trabajo específicos para estos perfiles laborales.

Desde el punto de vista de esta investigación, y teniendo en cuenta los numerosos beneficios que aporta esta práctica, se considera necesario continuar implementando programas de características similares e investigar su eficacia y efectividad, ya que no existen trabajos publicados al respecto sobre una población española. También se sugiere la posibilidad de plantear y diseñar un nuevo puesto de trabajo regulado para este nuevo perfil laboral.

Las limitaciones de esta investigación se relacionan con posibles experiencias de apoyo entre iguales en salud mental en España que no se hayan podido identificar. Además, no disponer de estándares en población española para esta práctica impide la comparación de experiencias con otras internacionales.

En definitiva, la educación es un campo emergente en el ámbito de la salud mental y en el cual se debe continuar diseñando experiencias y evaluando su impacto.

\section{Referencias bibliográficas}

Ariño, B. y San Pío, M. J. (2007). El papel de las asociaciones de familiares y personas con enfermedad mental. Revista Cuadernos de Psiquiatría Comunitaria, 7(2), 115-126.

Arnaiz, A. y Uriarte, J. J. (2006). Estigma y enfermedad mental. Norte de Salud Mental, 6(26), 49-59.

Asociación Española de Neuropsiquiatría (AEN) (2003). Rehabilitación psicosocial $y$ tratamiento integral del trastorno mental severo. Madrid: Asociación Española de Neuropsiquiatría. 
Barra, E. (2004). Apoyo social, estrés y salud. Psicología y salud, 14(2), 237-243.

Barrón, A. y Sánchez, E. (2001). Estructura social, apoyo social y salud mental. Psicothema, 13(1), 17-23.

Behavioral Health Work Group of the NPSCLN (2014). Advocating and planning for a behavioral health peer support program. North Caroline.

Bono del Trigo, A., Navarro, B. y Mena, A. (2011). Informe. Evaluación del proyecto ayuda mutua entrenamiento y prácticas en los servicios de salud mental ANDALUCÍA. Granada: Escuela Andaluza de Salud Pública.

Campos, F., Sousa, A., Rodrigues, V., Marques, A., Queirós, C. y Dores, A. (2016). Directrices prácticasparaprogramasdeapoyoentrepersonas con enfermedad mental. Revista de Psiquiatría y Salud Mental, 9(2), 97-110. https://doi. org/10.1016/j.rpsm.2014.06.002

Casas, L.A., López, L., Carrasco, O., Nieto-Moreno, M. y García-Heras, S. (2017). Profesional experto por experiencia en salud mental. XXVII Jornadas Estatales Salud Mental AEN: Madrid.

Chinman, M., McInnes, D. K., Eisen, S., Ellison, M., Farkas, M., Armstrong, M. y Resnick, S. G. (2017). Establishing a research agenda for understanding the role and impact of mental health peer specialists. Psychiatric Services, 68(9), 955-957. https://doi.org/10.1176/appi.ps.201700054

Confederación Salud Mental España (2017). Curso 'Informando en Primera Persona'. http://bit.ly/2oW9GyK

Convención Internacional de los Derechos de las Personas con Discapacidad. (2006). Adoptada por el Comité sobre los Derechos de las Personas con Discapacidad en la Sede de las Naciones Unidas en Nueva York el 13 de diciembre de 2006. (http:// www.un.org/esa/socdev/enable/documents/tccconvs.pdf)

Davidson, L. y González-Ibáñez, À. (2017). La recuperación centrada en la persona y sus implicaciones en salud mental. Revista de la Asociación Española de Neuropsiquiatría, 37(131), 189-205.

De Fuentes, C. (2016). La 'nueva' discapacidad mental. Revista Española de Discapacidad (REDIS), 4(1), 249-255. https:// doi.org/10.5569/2340-5104.04.01.15

Delman, J., Delman, D., Vezina, B. y Piselli, J. (2014). Peer led recovery learning communities: expanding social integration opportunities for people with the lived experience of psychiatric disability and emotional distress. Global Journal of Community Psychology Practice, 5(1), 1-11.

European Network on Independent Living (ENIL) (2018).ENIL and Peer support in 2016. http://bit.ly/2ECEZ7I

Fundación INTRAS et al. (2015). Peer2Peer. http://bit.ly/2sGBomi

Funk, M., Drew, N., Freeman, M. y Faydi, E. (2010). Salud mental y desarrollo: poniendo a las personas con problemas de salud mental como un grupo vulnerable. Ginebra: Organización Mundial de la Salud.

García-Pérez, O., Fernández García, C. M. e Inda Caro, M. M. (2017). Calidad de vida, alojamiento con apoyo y trastorno mental severo: el valor de la perspectiva socioeducativa en su recuperación. Revista Complutense de Educación, 28(2), 573-590. https://doi.org/10.5209/rev_RCED.2017. v28.n2.49633

García-Pérez, O., Peña-Calvo, J. V. y ToríoLópez, S. (2016). Atención socioeducativa y trastorno mental severo: la vivienda como base de intervención. Convergencia, 23(72), 171-197.

Gartner, A. J. y Riessman, F. (1982). Selfhelp and mental health. Psychiatric Services, 33(8), 631-635. https://doi. org/10.1176/ps.33.8.631

Gillard, S. y Holley, J. (2014). Peer workers in mental health services: literature overview. Advances in Psychiatric Treatment, 20(4), 286-292. https://doi.org/10.1192/ apt.bp. 113.011940

González, O. (2009). Enfermedad mental, 
estigma y legislación. Revista de la Asociación Española de Neuropsiquiatría, 29(2), 471-478.

Green, J., Willis, K., Hughes, E., Small, R., Welch, N., Gibbs, L. y Daly, J. (2007). Generating best evidence from qualitative research: The role of data analysis. Australian and New Zealand Journal of Public Health, 31(6), 545-550. https://doi. org/10.1111/j.1753-6405.2007.00141.x

Hernández, R. M. y Carranza, R. F. (2017). Felicidad, optimismo y autorrealización en estudiantes de un programa de educación superior para adultos. Interdisciplinaria, 34(2), 307-325. https://doi. org/10.16888/interd.2017.34.2.5

Intentional Peer Support (2017). What is IPS? https://bit.ly/2HYTT9L

Ley 26/2011, de 1 de agosto, de adaptación normativa a la Convención Internacional sobre los Derechos de las Personas con Discapacidad. «BOE» núm. 184, España, 2 de agosto del 2011.

Ley 39/2006, de 14 de diciembre, de Promoción de la Autonomía Personal y Atención a las personas en situación de dependencia. «BOE» núm. 299, España, 15 de diciembre del 2006.

Lloyd-Evans, B., Mayo-Wilson, E., Harrison, B., Istead, H., Brown, E., Pilling, S. y Kendall, T. (2014). A systematic review and meta-analysis of randomised controlled trials of peer support for people with severe mental illness. BMC, 14(1), 39. https://doi. org/10.1186/1471-244X-14-39

Lopera, I. y Echeverri, J. (2018). Libertad y desarrollo humano en las organizaciones. Interdisciplinaria, 35(2), 395-408. https://doi.org/10.16888/ interd.2018.35.2.9

López, L.A. (2008). Predictores del desempeño laboral de personas con discapacidad por trastorno mental severo: Revisión de estudios y análisis de evidencias. Psychosocial Intervention, 17(3), 245-268.

López, M., Fernández, L. y Laviana, L. (2010). Evaluación en entornos residenciales comu- nitarios. En J. A. Fernández, R. Touriño, N. Benítez y C. Abelleira (Eds.) Evaluación en rehabilitación psicosocial (pp. 195-212). Valladolid: FEARP.

López, M. y Laviana, M. (2017). Rehabilitación psicosocial y atención comunitaria: algunas consideraciones críticas y una propuesta de guion para el debate. Revista de la Asociación Española de Neuropsiquiatría, 37(131), 257-276.

López, M., Laviana, M., Fernández, L., López, A., Rodríguez, A. M. y Aparicio, A. (2008). La lucha contra el estigma y la discriminación en salud mental: Una estrategia compleja basada en la información disponible. Revista de la Asociación Española de Neuropsiquiatría, 28(1), 48-83.

Madoz-Gúrpide, A., Ballesteros, J. C., Leira, M. y García, E. (2017). Necesidad de un nuevo enfoque en la atención integral a los pacientes con trastorno mental grave treinta años después de la reforma psiquiátrica. Revista Española de Salud Pública, 91, 201701007.

Mead, S., Hilton, D. y Curtis, L. (2001). Peer support: A theoretical perspective. Psychiatric Rehabilitation Journal, 25(2), 134.

Mead, S. y MacNeil, C. (2006). Peer support: What makes it unique. International Journal of Psychosocial Rehabilitation, 10(2), 29-37.

Ministerio de Sanidad y Consumo (2007). Estrategia en Salud Mental del Sistema Nacional de Salud, 2006. Madrid: Autor.

Ministerio de Sanidad, Política Social e Igualdad (2011). Estrategia en Salud Mental del Sistema Nacional de Salud 2009-2013. Madrid: Autor.

Moreno, A. y Desviat, M. (2012). Del paternalismo a la ciudadanía. La dignidad y la libertad como elementos fundamentales del cuidado. En M. Desviat y A. Moreno (Eds.), Acciones de salud mental en la comunidad (pp. 787-796). Madrid: Asociación Española de Neuropsiquiatría.

Naslund, J. A., Aschbrenner, K. A., Marsch, L. A. y Bartels, S. J. (2016). The future of mental health care: peer-to-peer support and social media. Epidemiology and Psychia- 
tric Sciences, 25(2), 113-122. https://doi. org/10.1017/S2045796015001067

Organización Mundial de la Salud [OMS]. (2001). Informe sobre la salud en el mundo 2001: Salud mental: nuevos conocimientos, nuevas esperanzas. Ginebra: Autor.

Organización Mundial de la Salud [OMS]. (2004). Invertir en salud mental. Ginebra: Autor.

Organización Mundial de la Salud [OMS]. (2008). Programa de acción para superar las brechas en salud mental. Ginebra: Autor.

Organización Mundial de la Salud [OMS]. (2010). Salud mental y desarrollo: Poniendo el objetivo en las personas con problemas de salud mental como un grupo vulnerable. Ginebra: Autor.

Organización Mundial de la Salud [OMS]. (2015). Mental Health Atlas 2014. Ginebra: Autor.

Organización Mundial de la Salud [OMS]. (2017a). Creating peer support groups in mental health and related areas: WHO Quality Rights training to act, unite and empower for mental health (pilot version) (No. WHO/MSD/MHP/17.11). WHO.

Organización Mundial de la Salud [OMS]. (2017b). Helping people with severe mental disorders live longer and healthier lives: policy brief (No. WHO/MSD/MER/17.7). World Health Organization.

Organización Mundial de la Salud [OMS] (2018).WHO Quality Rights initiative - improving quality, promoting human rights. http://bit.ly/1iZaP1o

Orihuela, T., Conde, H. y Marcos, S. (2015). Peer2peer, Curso de formación profesional. Reino Unido: Scottish Recovery Network.

Ostrow, L. y Leaf, P. J. (2014). Improving capacity to monitor and support sustainability of mental health peer-run organizations. Psychiatric Services, 65(2), 239-241. https://doi.org/10.1176/appi.ps.201300187

Palomer, E., Izquierdo, R., Leahy, E., Masferrer, C. y Flores, P. (2010). El usuario como experto: concepto, modalidades y experiencia desde el Proyecto Emilia. Revista de la Asociación Española de Neuropsiquiatría, 30(1), 109-123.

Palomer, E., Izquierdo, R., Masferrer, C. y Flores, P. (2011). Advocacy: fomento y apoyo de la salud mental. Concepto, modalidades y agentes implicados. El proyecto Emilia como ejemplo. Átopos (Salud Mental, Comunidad y Cultura), 11, 5-17.

Pié, A. (2000). Intervenció educativa i salut mental. Educació social. Revista d'Intervenció Socioeducativa, (16), 91-104.

Real Decreto Legislativo 1/2013, de 29 de noviembre, por el que se aprueba el Texto Refundido de la Ley General de derechos de las personas con discapacidad y de su inclusión social.«BOE» núm. 289, España, 3 de diciembre del 2013.

Repper, J. y Carter, T. (2011). A review of the literature on peer support in mental health services. Journal of Mental Health, 20(4), 392-411.

Repper, J., Aldridge, B., Gilfoyle, S., Gillard, S., Perkins, R. y Rennison, J. (2013). Peer support workers: Theory and practice. London: Centre for Mental Health.

Robles, E. (2017). Servicios de asistencia personal para personas con problemas de salud mental. Observatorio de Modelos Integrados en Salud de New Health Foundation. https://bit.ly/2W7frbT

Rodríguez, A. (2007). Rehabilitación psicosocial y atención social comunitaria a las personas con enfermedades mentales crónicas en la Comunidad de Madrid. En F. Lezcano y M. A. Martínez (Coords.), Conocer y aceptar: el reto de trabajar con personas con enfermedad mental grave (pp. 83-131). Burgos: Caja de Burgos.

Ruiz, M. A., Montes, J. M., Correas, J., Álvarez, C., Mauriño, J. y de Dios, C. (2012). Opiniones y creencias sobre las enfermedades mentales graves (esquizofrenia y trastorno bipolar) en la sociedad española. Revista de Psiquiatría y Salud Mental, 5(2), 98-106. https://doi. org/10.1016/j.rpsm.2012.01.002 
Smith, L. y Bradstreet, S. (2011). Experts by experience: guidelines to support the development of Peer Worker roles in the mental health sector. Glasgow: Scottish Recovery Network.

Torras, A. (2012). Visibilidad, protesta y denuncia. Algunas propuestas. En M. Desviat y A. Moreno (Eds.), Acciones de salud mental en la comunidad (pp. 835-848). Madrid: Asociación Española de Neuropsiquiatría.

Tortella-Feliu, M., Baños, R., Barrantes, N., Botella, C., Fernández-Aranda, F., García-Campayo, J., García-Palacios, A., Hervás, G., Jiménez-Muercia, S., Montorio, I., Soler, M., Quero, S., Valiente, M.C. y Vázquez, C. (2016). Retos de la investigación psicológica en salud mental. Clínica y Salud, 27(1), 37-43. https://doi.org/10.1016/j.clysa.2016.02.001

University of Nebraska (2013). Nebraska Peer Support Certification Study. January 2014. Lincoln: University of Nebraska.

Vega-Angarita, O. M. y Gonzalez-Escobar, D. S. (2017). Apoyo social: Elemento clave en el afrontamiento de la enfermedad Crónica. Ciencia y Cuidado, 6(1), 51-62. https://doi.org/10.6018/eglobal.8.2.66351

Watson, E., Lambert, M. y Machin, K. (2016). Peer support training: values, achievements and reflections. Mental Health Practice, 19(9), 21-27. https://doi.org/10.7748 / mhp.19.9.22.s20

Recibido: 8 de noviembre de 2019 Aceptado: 4 de marzo de 2021 